\title{
MIS Posterior Cervical Spine Surgery: Five- Level Fusion through a Novel Cervical Tube
}

\author{
Ashwini D. Sharan, MD', Mitchell A. Hardenbrook, MD², John Ratliff, MD', \\ James S. Harrop, MD \\ ${ }^{1}$ Department of Neurological Surgery, Jefferson Medical College, Philadelphia, PA \\ ${ }^{2}$ The Boston Spine Group, Newton, MA
}

\section{Introduction}

Minimally invasive surgical (MIS) techniques to the spine have focused mainly on the lumbar region. Many reports exist on the ability to decompress the neural structures in the lumbar and thoracic spine both via an anterior and posterior approach. ${ }^{1-4}$ Familiarity with these techniques now allows one or two level interbody and pedicle fusions, and treatment of trauma through MIS approaches., Controversy exists as to the efficacy of these techniques because direct studies comparing MIS approaches with open techniques are lacking. However, proponents of these techniques site smaller incision, less muscle retraction, less blood loss, shorter length of stay and better recovery.

As such, few reports exist on the use of MIS techniques in the cervical spine. Clearly, the anatomic constraints of the cervical spine are different, but they arguably lend themselves more amenable to MIS approaches. We present two cases in which five-level posterior cervical fusion was achieved using a tubular retractor specifically designed for the cervical spine.

\section{Case 1}

The patient is a 35-year old male with a past medical history of intravenous heroin abuse who presented with a two-month history of neck stiffness and tenderness. The patient also had paresthesias that began in his left hand and then progressed into both upper extremities, chest, and descended down through the torso into the legs. The patient had also noticed distal hand weakness for about two weeks. He had a three-day history of difficulty ambulating and leg weakness. There were no antecedent fevers or chills or other manifestations of infection or injury. He was transferred from another institution with a report of a C6 vertebral fracture with an epidural lesion.

Upon admission, his strength in the upper extremities was $3 / 5$ motor strength in his triceps, grip, and intrinsic hand strength. He had approximately $4 / 5$ strength in both lower extremities. He had a C6 sensory level to pin prick. He was hyperreflexive in both lower extremities with bilateral Babisnki's sign.

Laboratory studies were positive for a high ESR $=93(0-10)$ and $\mathrm{CRP}=1.30(0.0-0.8)$ and $\mathrm{WBC}=$ 7.4 (4-11.0). His work up included plain X-rays of the cervical spine, which were limited because of shoulder girth. A CT Scan and MR image of the cervical spine with and without gadolinium revealed an epidural abscess and vertebral osteomyelitis and discitis with partial destruction of the C6 and C7 vertebral bodies and kyphotic deformity (Figures 1).

The patient went to the operating room and had an anterior C6 and partial C7 corpectomy, correction of deformity, and arthrodesis with iliac crest autograft bone. In a second stage, the patient underwent an MIS posterior cervical stabilization with lateral mass screws at $\mathrm{C} 4, \mathrm{C} 5, \mathrm{C} 7$ and pedicle screws at $\mathrm{T} 1$ using a cervical tubular retractor (Endius, Inc) (Figures 2 and 3). The operative cultures were positive for methacillin-resistant staphylococcus aureus (MRSA). Post-operatively, the patient was treated with 6 weeks of intravenous antibiotics and 6 weeks of oral antibiotics. Six months later, the patient is infection free and neck pain free (Figure 4 ). His neurological status improved while in the hospital and he was discharge to home.

\section{Case 2}

The patient is a 58-year old male with a 15 -month history of neck pain radiating to left arm. MRI revealed C4-T1 left foraminal stenosis and central stenosis at C5-6. The patient was treated with five-level (C3-T1) instrumented posterior spinal fusion, C3-4, C4-5, C5-6, C6-7, C7-T1 left hemilaminotomies and C5-6 laminectomy through a $6.5 \mathrm{~cm}$ incision.

\section{Discussion}

Lateral mass plating with screws were first described by Roy-Camillle. ${ }^{1-4}$ Their technique provided immediate stability of the cervical spine and was feasible even when the lamina and spinous processes were damaged. The procedure was further modified and developed by Magerl. ${ }^{5}$ All these techniques focused on safe screw placement on the basis of anatomical landmarks and trajectories to avoid the nerve roots, the spinal cord, and the vertebral artery. Nevertheless, these traditional approaches required large midline incisions with stripping of the paraspinal muscles in the midline and resulted in significant postoperative neck pain.

Roh in 2000 advocated that the MED technique allowed a better decompression compared with the standard open technique in four cadaveric specimens. ${ }^{6}$ Adamson performed a microendoscopic posterior cervical lamiforaminotomy for unilateral radiculopathy on 100 patients and had excellent or good results in 97 of them with no serious complications reported. ${ }^{7}$ Use of the MED technique in posterior cervical discectomy and foraminotomy showed excellent results with minimal disadvantages.

Percutaneous cervical screws fixation is a novel surgical technique. There are few cases reported in the literature..$^{8-11}$ Wang reported on 3 cases of single-level posterior cervical fusion on traumatic instability achieved through a METRx ${ }^{\circ}$ tube (Medtronic Sofamor Danek, Memphis, TN). ${ }^{9}$ Fong et al. reported on two additional patients with trauma requiring a posterior cervical tension band through a METRx ${ }^{\otimes}$ tube. ${ }^{11}$ In that same report, they showed that in a cadaveric model, a four level posterior cervical spinal fusion could be theoretically achieved while "wanding" the retractor to allow rostralcaudal visualization. Joseffer et al. described the use of the Quadrant ${ }^{\text {Tn }}$ retractor (Medtronic Sofamor Danek, Memphis, TN) to deliver unilateral C1-2 screws for fixation of an Os Odontoideum. ${ }^{10}$

The largest series on MIS posterior cervical instrumentation is described by Wang et al. ${ }^{8}$ They retrospectively reviewed their first 18 patients treated with this technique with two year follow-up. Half of their patients were 


\section{Spine}

treated with a single level and half with two-level. Additionally, six had unilateral instrumentation and the remainder had bilateral. There were no hardware failures. Blood loss averaged $112 \mathrm{ml}$.
Two patients in the series required conversion to the open technique because of inability to obtain adequate visualization with fluoroscopy through the bulky shoulders.
We were able to obtain MIS posterior cervical fusion across five levels using the Endius NexPosure ${ }^{\text {Ta }}$ tubular retractor (Endius Inc, Plainville, MA). This tube was designed specifically
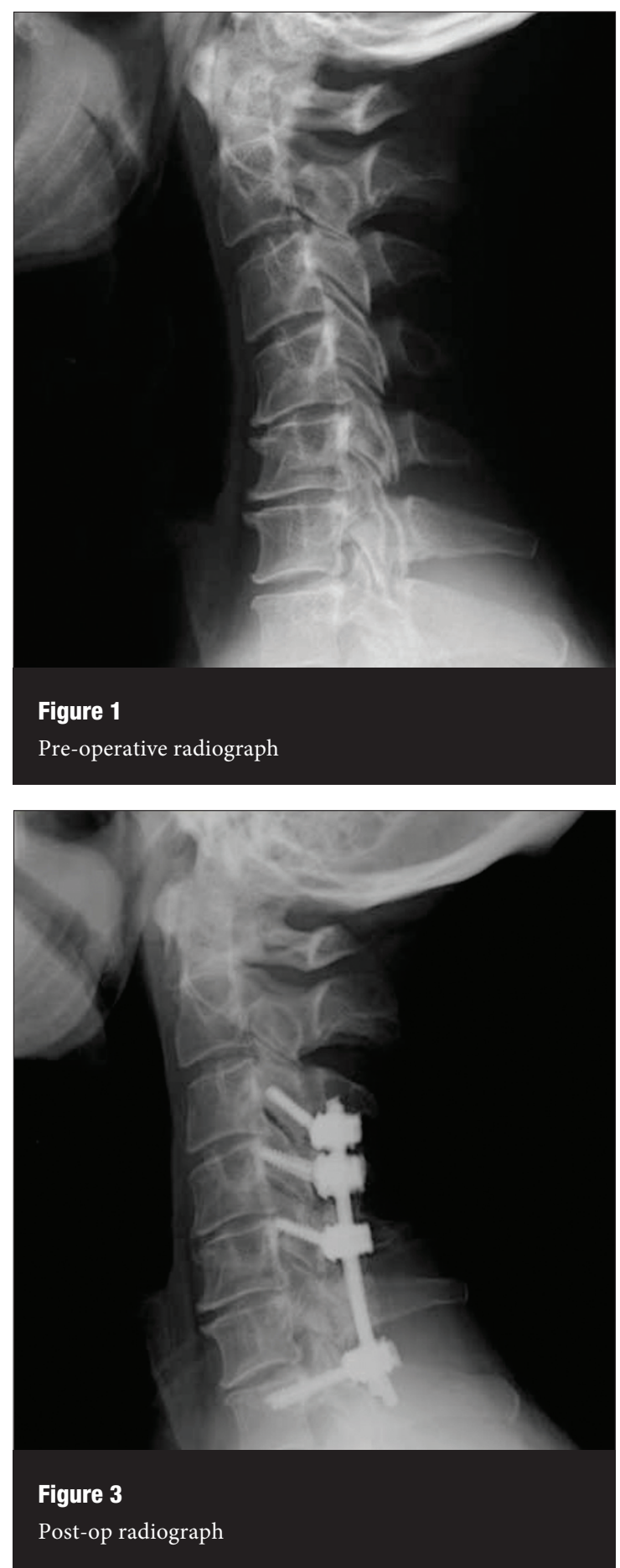

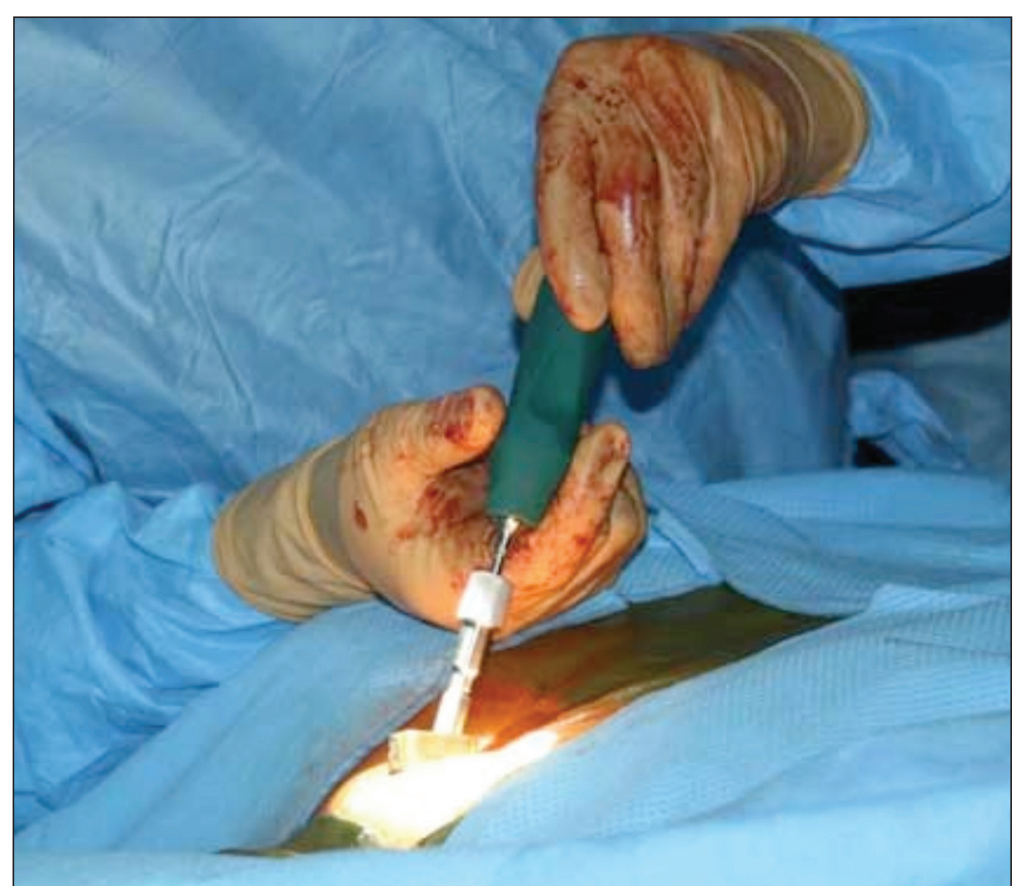

Figure 2

Intraoperative photo

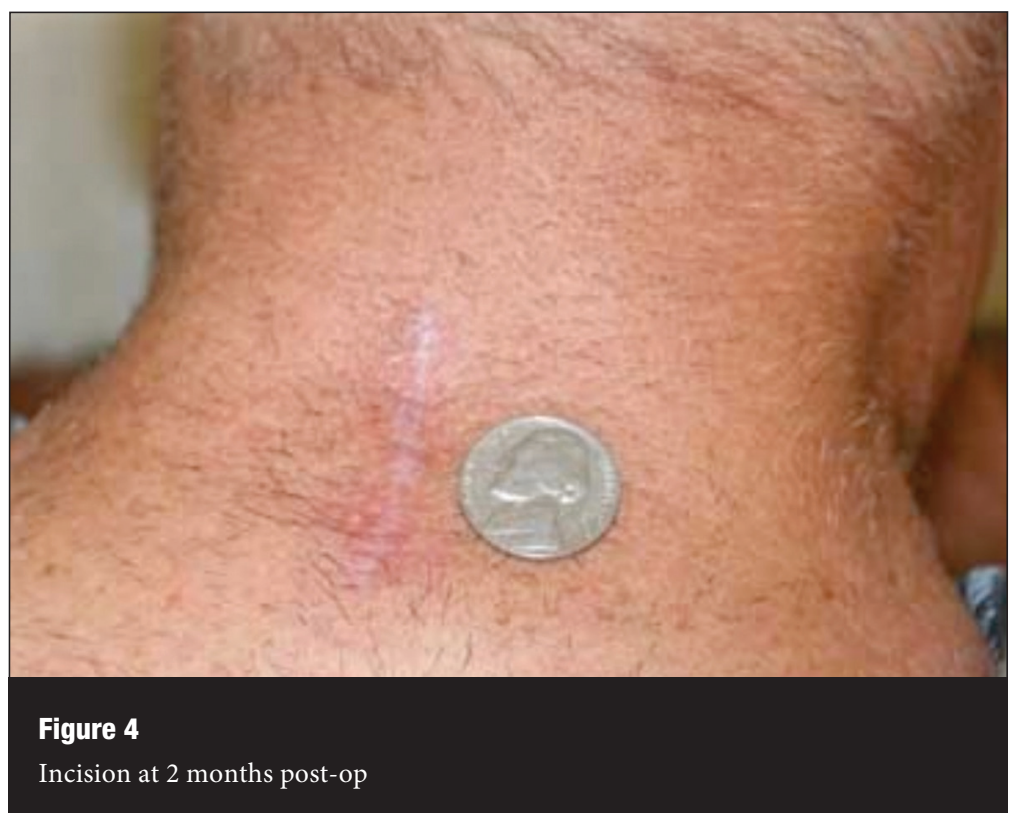


for access to the cervical spine. The tube is engineered such that there is a medial cut-out in the skirt which facilitates docking of the tube onto the cervical lamina which is angled up dorsally from the facet. Additionally, the tube employs the ability to provide both rostral-caudal as well as medial-lateral angulation. This latter feature greatly facilitates the insertion of both sub-axial lateral mass screws laterally, and C7 or T1 pedicle screws medially. Finally, the expanded skirt permits larger subfascial exposure with a smaller skin incision.

This technique has several advantages. Mainly, the incision size is limited and attempts are made to preserve the musculature attachments to the midline. It is felt that this will reduce postoperative pain. ${ }^{9,12}$ However, there are also some limitations. The working space is narrow due to the small diameter of the tubular retractors, the rod placement through the retractor can be technically difficult with the retractors available at this time, and it is technically challenging to instrument more than four adjacent levels. Relevant bony landmarks can be visualized through the tube. There is the usual learning curve with a new technique and the operator must get used to visualizing both medially and laterally though the tube; medially to correctly identify the lamina facet border and laterally to avoid an external breech of cortical bone with the screw.

\section{Technique}

The patient is placed in the prone position with the head rigidly fixed in a 3-pin head holder while keeping the cervical spine in a neutral posture. A guide wire is used with fluoroscopy to determine the ideal position for the skin incision. A sagittal trajectory parallel to the facet joint is desired and the entry point on the skin should be at the midline two to three levels below the desired level. A midline incision is made and then two parallel incisions are made through the fascia just off the midline. The opening through the fascia will have to be extended more rostrally and caudally to accommodate the diameter of the tubular retractors. The operator may have to remove the lateral aspects of a bifid spinous process should this force the incision too laterally.

\section{References}

1. Roy-Camille, M., G. Saillant, and D. verteaux, Early management in spinal injuries, in Recent Advances in Orthopedics., $\mathrm{B}$. McKibbon, Editor. 1979, Churchill-Livingstone: Edinburgh. p. $57-87$.

2. Roy-Camille, R., M. Roy-Camille, and C. Demeulenaere, [Osteosynthesis of dorsal, lumbar, and lumbosacral spine with metallic plates screwed into vertebral pedicles and articular apophyses]. Presse Med, 1970. 78(32): p. 1447-8.

3. Roy-Camille, R., et al., [Recent injuries of the last 5 cervical vertebrae in the adult (with and without neurologic complications)]. Sem Hop, 1983. 59(19): p. 1479-88.

4. Roy-Camille, R., et al., Treatment of lower cervical spinal injuries-C3 to C7. Spine, 1992. 17(10 Suppl): p. S442-6.
5. Magerl, F., D. Grob, and P. Seeman, Stable dorsal fusion of thye cervical spine (C2-T1) using hook plates, in Cervical Spin I, P. Kehr and A. Weidner, Editors. 1987, Springer-Verlag.: Strasbourg. p. 217-221.

6. Roh, S.W., et al., Endoscopic foraminotomy using MED system in cadaveric specimens. Spine, 2000. 25(2): p. 260-4.

7. Adamson, T.E., Microendoscopic posterior cervical laminoforaminotomy for unilateral radiculopathy: results of a new technique in 100 cases. J Neurosurg, 2001. 95(1 Suppl): p. 51-7.

8. Wang, M.Y. and A.D. Levi, Minimally invasive lateral mass screw fixation in the cervical spine: initial clinical experience with long-term follow-up. Neurosurgery, 2006. 58(5): p. 907-12; discussion 907-12.

9. Wang, M.Y., et al., Minimally invasive lateral mass screws in the treatment of cervical facet dislocations: technical note. Neurosurgery, 2003. 52(2): p. 444-7; discussion 447-8.

10. Joseffer, S., et al., Minimially invasive atlantoaxial fixation with a ployaxial screw-rod construct: technical case report. Neurosurgery, 2006. 58[ONS Suppl 2]: p. 375.

11. Fong, S.Y. and S. Duplessis, Minimally Invasive Lateral Mass Plating in the Treatment of Posterior Cervical Trauma: Surgical Technique. J Spinal Disord Tech, 2005. 18(3): p. 224-228.

12. Shiraishi, T., A new technique for exposure of the cervical spine laminae. Technical note. J Neurosurg, 2002. 96(1 Suppl): p. 122-6.

\section{Corresponding Author}

Ashwini D. Sharan, MD

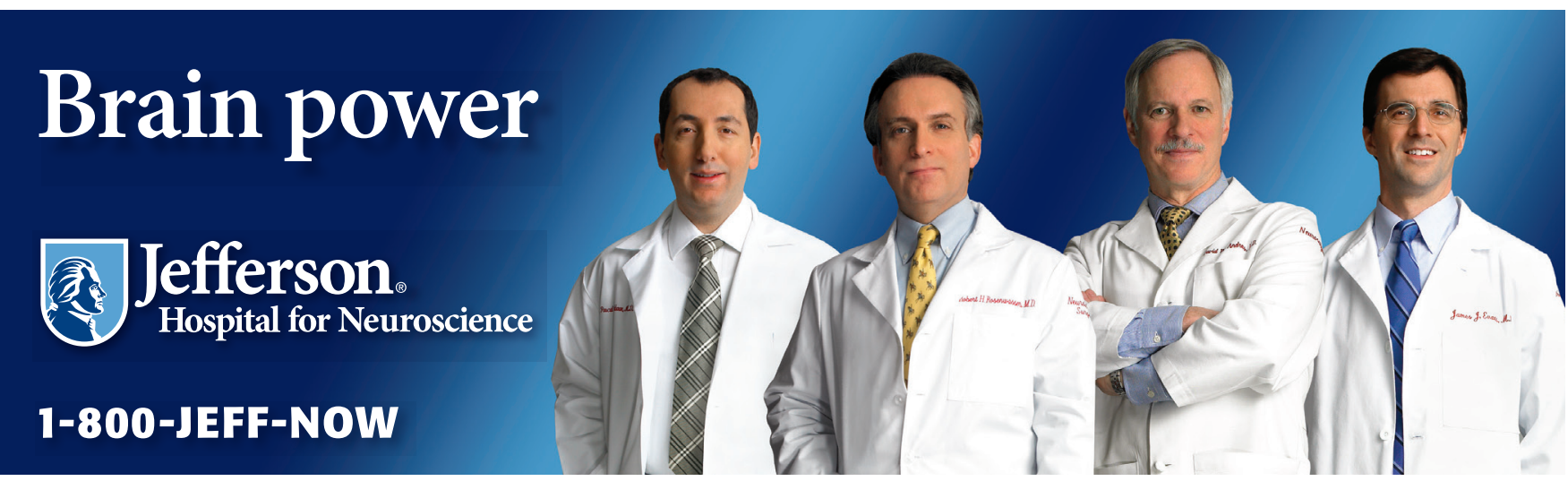

